\title{
Clinical Pattern of Recurrent Disease during the Follow-Up of Rectal Carcinoma
}

\author{
Thijs Wieldraaijer $^{\mathrm{a}}$ Pascal Bruin ${ }^{\mathrm{a}}$ Laura A.M. Duineveld ${ }^{\mathrm{a}}$ Pieter J. Tanis ${ }^{\mathrm{b}}$ \\ Anke B. Smits ${ }^{c}$ Henk C.P.M. van Weert ${ }^{\mathrm{a}}$ Jan Wind ${ }^{\mathrm{a}}$ \\ ${ }^{a}$ Department of Primary Care, Academic Medical Centre, and ${ }^{b}$ Department of Surgery, Academic Medical Centre, \\ Amsterdam, and ${ }^{\mathrm{C} D e p a r t m e n t}$ of Surgery, St. Antonius Hospital Nieuwegein, Nieuwegein, The Netherlands
}

\section{Keywords}

Rectal cancer · Recurrent disease · Patients · Follow-up ·

Clinical presentation

\begin{abstract}
Background/Aims: Several initiatives have started to transfer colorectal cancer follow-up (FU) from secondary to primary care. For this purpose, it is important to assess when and how recurrences of rectal carcinoma are detected after treatment with curative intent. Methods: Retrospective multicentre cohort study. Patients participating in an FU programme after curative intended treatment for rectal cancer stages I-III between 2007 and 2014. Results: Of the 378 patients, 64 (17\%) developed recurrent disease (RD). Most were detected during scheduled FU consultations ( $n=55$ ) by (a combination of) radiological examinations and carcinoembryonic antigen levels, and were asymptomatic $(n=53)$; outside scheduled FU consultations, RD was detected during the treatment of postoperative complications or ostomy reversal $(n=5)$, or due to symptoms $(n=4)$. Most frequent sites of recurrence were liver (50\%), lung (44\%), multiple (22\%) or locoregional (16\%). Treatment of RD with curative intent was performed more frequently when detected during scheduled FU (60 vs. $22 \%$ ). The only predictive factor for developing RD was stage III disease on initial presentation. Conclusions: The majority of rectal cancer patients are diag-
\end{abstract}

\section{KARGER}

E-Mail karger@karger.com www.karger.com/dsu
Karger

Open access
This article is licensed under the Creative Commons AttributionNonCommercial-NoDerivatives 4.0 International License (CC BYNC-ND) (http://www.karger.com/Services/OpenAccessLicense). Usage and distribution for commercial purposes as well as any distribution of modified material requires written permission.

nosed with RD at an asymptomatic stage during scheduled FU consultations. Only a few patients presented with RD outside theFU programme. Arguably, general practitioners could order these same diagnostic tests during FU.

(C) 2017 The Author(s)

Published by S. Karger AG, Basel

\section{Introduction}

Colorectal cancer (CRC) is one of the most common types of cancer, with more than 15,000 new cases in the Netherlands in 2015 [1]. About 30\% of all cases of CRC is located in the rectum [1]. The cornerstone of intentionally curative treatment of rectal carcinoma is surgical resection, preceded by (chemo) radiotherapy if indicated. Five-year overall survival rate currently approximates $65 \%$ [1-3]. Following primary treatment, patients are included in a follow-up (FU) programme to provide supportive care in the years following treatment and to detect recurrent disease (RD) at an asymptomatic stage, since this has been shown to improve treatment options [4-9]. Rectal cancer FU typically consists of periodic consultations with laboratory and radiological examinations. The most optimal FU schedule has not been defined, and FU is generally outlined by national guidelines with some inter-hospital variability [2].

Thijs Wieldraaijer, MD

Department of Primary Care AMC-UvA Post Box 22660

NL-1100 DD Amsterdam (The Netherlands)

E-Mail t.wieldraaijer@amc.uva.nl 
Table 1. Rectal cancer follow-up schedule ${ }^{1}$

\begin{tabular}{|c|c|c|c|c|}
\hline & Year 1 & Year 2 & Year 3 & Year 4-5 \\
\hline Consultation & Every 6 months & Every 6 months & Every 6 months & Yearly \\
\hline CEA blood test (cut-off point $\geq 5.5 \mu \mathrm{g} / \mathrm{L}$ ) & Every 3-6 months & Every 3-6 months & Every 3-6 months & Every 6 months \\
\hline Imaging of liver (ultrasound or CT abdomen ${ }^{2}$ ) & Every 6 months & Every 6 months & Yearly & Yearly \\
\hline Colonoscopy & After 1 year ${ }^{4}$ & & $\begin{array}{l}\text { Depending on } \\
\text { previous } \\
\text { colonoscopy: after } \\
3-5 \text { years }\end{array}$ & \\
\hline
\end{tabular}

${ }^{1}$ In pT1N0 tumours, CEA and imaging are optional. ${ }^{2}$ If ultrasound is unreliable or patient has high risk of recurrence. ${ }^{3}$ If patient
has a high risk of recurrence. ${ }^{4}$ If there was no (complete) preoperative colonoscopy it should be performed 3 months after surgery.

Increasing incidence, improved treatment, and the recently started national screening programme to detect CRC in the Netherlands will lead to a rise in the number of patients in FU $[1,2,10]$. This rise might further congest hospital outpatient clinics, compromising the ability to offer comprehensive FU care, including attention to psychosocial effects of cancer and its treatment, and frequent comorbid conditions [10-12]. Because general practitioners (GPs) are more accustomed to offering such comprehensive care, several initiatives have proposed to transfer FU from secondary to primary care [2, $10,11]$.

While few studies assessed the feasibility of transferring colon cancer FU to primary care $[13,14]$, no such research has been performed for rectal cancer.

The first step towards transfer of FU to primary care, and the purpose of this study, was to assess when and how $\mathrm{RD}$ was detected - whether this was during scheduled FU consultations, by means of additional diagnostic examinations, and whether or not patients presented with symptoms.

\section{Methods}

\section{Study Design and Outcome}

We performed a retrospective cohort study to determine when $\mathrm{RD}$ of rectal carcinoma was discovered, which tests identified RD, whether RD was detected during a scheduled FU visit or an interval consultation and if there were symptoms present suggestive for RD.
Patients

Patients were selected from 2 hospitals in the Netherlands: the Academic Medical Centre (AMC) in Amsterdam, and the St. Antonius Hospital in Nieuwegein. Using surgery record lists for the AMC from 2007 until 2009 and the national colorectal registration database (the Dutch surgical colorectal audit) for both hospitals from 2009 until 2014, all patients who underwent surgery for rectal cancer were selected. Inclusion criteria were treatment with curative intent for a primary rectal carcinoma stage I, II or III, defined as a tumour located within 15 centimetres of the anal verge. Exclusion criteria were: stage IV disease, local excision (transanal endoscopic microsurgery), hereditary colorectal carcinoma, colorectal carcinoma in the presence of inflammatory bowel disease, or synchronous malignancies other than rectal carcinoma. Additionally, in-hospital deaths following surgery, or patients not eligible for scheduled FU were excluded from analysis.

\section{FU Schedule}

FU in both hospitals is performed according to the Dutch national guideline $[2,3]$, which is depicted in Table 1 . FU is generally coordinated by surgeons. In the St. Antonius Hospital, it is commonly an oncologist who coordinates FU after adjuvant therapy.

\section{Data Extraction}

Data was retrieved by studying the electronic patient records of all included patients in both hospitals. Baseline characteristics were documented: tumour stage, site of tumour, therapy, radical extent of surgery, FU consultations and examinations, and whether patients developed RD during the FU period. An FU examination was coded as "missing" if it did not take place, or was carried out more than 3 months later than the period that the guideline suggests.

When a patient was diagnosed with locoregional recurrence, metachronous colorectal carcinoma or distant metastases, additional information was extracted; whether patients were diagnosed by examinations performed as part of scheduled FU or not, which diagnostic test(s) resulted in the discovery of $\mathrm{RD}$, and the site of 
$\mathrm{RD}$. If symptoms led to the discovery of $\mathrm{RD}$, or if patients had symptoms that could be related to RD at the time of diagnosis, they were labelled "symptomatic." Furthermore, proceedings after diagnosis with RD were documented: treatment with curative intent, palliative treatment with chemotherapy or radiotherapy (RT), or supportive care. All data of patients with RD were checked by 2 researchers (T.W. and P.B.). In case of disagreement, a third researcher (J.W.) was consulted.

\section{Statistics}

Statistical calculations were performed using SPSS version 23.0 package (SPSS, Chicago, IL, USA). $\chi^{2}$ and Fisher's exact test were used to compare group means and predictive factors for RD. The Kaplan-Meier analysis was used to calculate the 5 -year overall survival and recurrence-free survival for all patients, and for survival after detection of RD. The date of the surgical resection of the primary rectal cancer was used as the starting point for survival analysis. The log-rank test was used for comparison of recurrences detected during regular FU and in between consultations. Patients who were lost to FU were censored in the analysis.

\section{Results}

\section{Patients}

During the studied period, 550 patients underwent surgery for rectal cancer of whom 172 were excluded (Table 2). The remaining 378 patients had a median FU of 30 months (interquartile range [IQR] 15-49). Neoadjuvant therapy was given in 317 (84\%) patients; this therapy consisted of chemoradiotherapy in 143 (45\%). Characteristics are shown in Table 3 . At the time of data extraction, 62 patients had completed 5 years of FU, 227 patients were still participating in FU, 13 patients were lost to FU and 76 patients stopped prematurely with scheduled FU: because of RD $(n=64)$, patient's request for termination $(n=4)$, age $(n=4)$, comorbidity $(n=3)$ or transfer of FU to another hospital $(n=1)$.

In total, 218 patients (58\%) had received all examinations recommended by the national guideline: 249 patients (66\%) received all carcinoembryonic antigen (CEA) blood tests according to the planned FU schedule, 63 patients (17\%) missed 1 test, and 66 patients (17\%) missed 2 or more tests. Three hundred and eight patients (82\%) received all planned radiological examinations (ultrasound, CT or MRI), 53 patients (14\%) missed 1 test, and 17 patients $(4 \%)$ missed 2 or more tests.

Of the 165 patients with over 3 years of FU and no RD, colonoscopy within 3 years after primary surgery was performed in 111 patients (67\%). Whether there was a specific reason for not performing a surveillance colonoscopy, for example, at the patient's request, was not always clear from the hospital records.

Clinical Pattern of RD during FU of Rectal Carcinoma
Table 2. Excluded patients $(n=172)$

\begin{tabular}{|c|c|c|}
\hline Reason & Number & $\begin{array}{l}\text { Percentage of } \\
\text { reasons for } \\
\text { exclusion }\end{array}$ \\
\hline Stage IV disease & 49 & 28 \\
\hline IBD & 30 & 17 \\
\hline Other primary carcinoma ${ }^{1}$ & 28 & 16 \\
\hline No scheduled follow-up ${ }^{2}$ & 24 & 14 \\
\hline In-hospital death & 18 & 10 \\
\hline Hereditary rectal carcinoma & 17 & 10 \\
\hline Other primary carcinoma & 6 & 3 \\
\hline Recurrence of rectal carcinoma & 3 & 2 \\
\hline TEM & 3 & 2 \\
\hline
\end{tabular}

IBD, inflammatory bowel disease; TEM, transanal endoscopic microsurgery. ${ }^{1}$ Synchronous tumour(s) other than rectal carcinoma. ${ }^{2}$ Because of return to hospital close to patient's residence $(n=8)$, age $(n=6)$, without reason $(n=5)$, comorbidity $(n=2)$, nursing home $(n=2)$, patient's request $(n=1)$.

Table 3. Characteristics of included patients $(n=378)$

\begin{tabular}{lc}
\hline & $n(\%)$ \\
\hline Age, median (IQR) & $66(58-72)$ \\
Male & $260(69)$ \\
Neoadjuvant RT & $17(84)$ \\
$\quad$ Short & $317(46)$ \\
$\quad$ Chemoradiotherapy (short) & $5(1)$ \\
Chemoradiotherapy (long) & $138(37)$ \\
Site of cancer & \\
Distal $(0-5 \mathrm{~cm})$ & $186(49)$ \\
Mid $\left(6-10 \mathrm{~cm}^{2}\right)$ & $138(37)$ \\
Proximal (11-15 cm) & $54(14)$ \\
Stage(p) & \\
pCR & $20(5)$ \\
I & $134(35)$ \\
II & $87(23)$ \\
III & $137(36)$ \\
Type of surgery & \\
Open LAR & $54(14)$ \\
Lap LAR & \\
Open APR & $169(45)$ \\
Lap APR & $53(14)$ \\
Radical surgical resection & \\
Adjuvant CHT & \\
\hline
\end{tabular}

PCR, pathological complete response. ${ }^{1}$ Radiotherapy short course $(5 \times 5 \mathrm{~Gy})$ or long course $(25 \times 2$ or $28 \times 1.8 \mathrm{~Gy}) .{ }^{2}$ Distance from anal verge. ${ }^{3}$ Pathological stage. ${ }^{4}$ Open or laparoscopic (Lap), low anterior resection (LAR) or abdominoperineal resection (APR). ${ }^{5}$ Missing data $(n=6) .{ }^{6}$ Adjuvant chemotherapy frequently until 2010, not a part of treatment since 2011. 


\section{Recurrences of Rectal Carcinoma}

Sixty-four patients (17\%) were diagnosed with RD, of whom 55 (86\%) were detected during scheduled FU visit. In the remaining 9 patients (14\%), RD was detected during non-scheduled (interval) consultations. Five were isolated locoregional recurrences and 5 included locoregional recurrences. The median time till detection of RD was 15 months (IQR 9-25). Confirmation of recurrence was done with imaging or biopsy. Of all recurrences, $43 \%$ were detected within the first year after surgery, $77 \%$ within 2 years, and $97 \%$ within 3 years. Two patients were diagnosed with $\mathrm{RD}$ after more than 3 years of FU.

A comparison of patients that developed $\mathrm{RD}$ with those that did not is shown in Table 4. The only independent predictive factor for developing RD was stage III carcinoma $(p<0.001)$. There was no association between missing FU consultations or examinations and detection of RD.

Sites of recurrence were most frequently the liver $(n=$ $32 ; 50 \%)$ and lungs $(n=28 ; 44 \%)$. RD had multiple localisations in 14 patients (22\%; Table 5). Re-operation with curative intent was performed in 35 patients (55\%) with $\mathrm{RD}$, while 23 patients (36\%) started palliative treatment soon after diagnosis. RD was detected at a symptomatic stage in 6 patients (9\%): 4 patients with locoregional recurrences and 2 with distant metastases. None of the symptomatic patients were treated with curative intent because of advanced disease, and 5 patients started palliative treatment shortly after RD was detected.

\section{$R D$ Detected during Scheduled FU}

In 55 patients, $\mathrm{RD}$ was detected during scheduled FU, with 53 patients (96\%) being asymptomatic. Imaging raised suspicion of $\mathrm{RD}$ in 26 patients (49\%): by means of CT-scan $(n=10)$, ultrasonography $(n=8)$ or chest radiography $(n=8)$. Elevated CEA levels raised the suspicion of the presence of RD in 18 patients (34\%) with a median value of $11.3 \mu \mathrm{g} / \mathrm{L}$ (median increase: $5.5 \mu \mathrm{g} / \mathrm{L}$ ). Both radiological and CEA levels simultaneously raised suspicion in 8 patients (15\%). RD was detected by colonoscopy in 1 patient. Two symptomatic patients presented themselves on scheduled FU consultations with 1 patient having a palpable tumour during a painful digital rectal examination and the other patient having lymphedema of the left leg.

Of the patients with RD detected during scheduled FU, 33 patients $(60 \%)$ were re-operated with curative intent, whereas 16 (29\%) started palliative treatment soon after diagnosis.
Table 4. Comparison of patient characteristics: recurrences versus no recurrences

\begin{tabular}{|c|c|c|c|}
\hline & $\begin{array}{l}\text { No } \\
\text { recurrences }\end{array}$ & Recurrences & $p$ value \\
\hline Patients, $n(\%)$ & $314(83)$ & $64(17)$ & \\
\hline Age, median (IQR) & $66(58-73)$ & $65(57-70)$ & 0.099 \\
\hline Gender, male, $n(\%)$ & $216(69)$ & $44(69)$ & 0.995 \\
\hline Site of cancer ${ }^{1}, n(\%)$ & & & 0.679 \\
\hline Distal $(0-5 \mathrm{~cm})$ & $153(49)$ & $33(52)$ & \\
\hline $\operatorname{Mid}(6-10 \mathrm{~cm})$ & $114(36)$ & $24(38)$ & \\
\hline Proximal $(11-15 \mathrm{~cm})$ & $47(15)$ & $7(11)$ & \\
\hline Stage $(\mathrm{p})^{2}, n(\%)$ & & & $<0.001$ \\
\hline $\mathrm{pCR}^{3}$ & $19(6)$ & $1(2)$ & \\
\hline I & $125(40)$ & $9(14)$ & \\
\hline II & $79(25)$ & $8(13)$ & \\
\hline III & $91(29)$ & $46(72)$ & \\
\hline Type of surgery ${ }^{4}, n(\%)$ & & & 0.971 \\
\hline Open LAR & $44(14)$ & $10(16)$ & \\
\hline Lap LAR & $140(45)$ & $29(45)$ & \\
\hline Open APR & $45(14)$ & $8(13)$ & \\
\hline Lap APR & $85(27)$ & $17(27)$ & \\
\hline Radical surgical & & & \\
\hline resection ${ }^{5}, n(\%)$ & $298(95)$ & $59(92)$ & 0.724 \\
\hline
\end{tabular}

${ }^{1}$ Distance from anal verge. ${ }^{2}$ Pathological stage. ${ }^{3}$ Pathological complete response. ${ }^{4}$ Open or laparoscopic (Lap), low anterior resection (LAR) or abdominoperineal resection (APR). ${ }^{5}$ Missing data $(n=6)$.

Table 5. Localisation recurrence/metastasis $(n=64)$

\begin{tabular}{lc}
\hline Site & $n(\%)$ \\
\hline Multiple $^{1}$ & $14(22)$ \\
Liver (isolated) & $32(50)$ \\
Lung (isolated) & $28(44)$ \\
Locoregional & $10(16)$ \\
Anastomosis & $3(5)$ \\
Pelvic & $7(11)$ \\
Lymph nodes & $9(14)$ \\
Peritoneal & $3(5)$ \\
Other & $2(3)$ \\
\hline
\end{tabular}

${ }^{1}$ A combination of sites listed in this table. ${ }^{2}$ Pancreas, skeletal, subcutaneous and adrenal gland.

\section{RD Detected Outside Scheduled FU Visits}

In 9 patients, RD was detected outside the scheduled FU visits; of the 9, 4 had symptoms.

Symptoms that led to detection of recurrence were jaundice, weight loss, abdominal pain with fever and 
strangury. Three of the 5 asymptomatic recurrences were identified during the treatment of a postoperative abdominal abscess (with metastases observed in liver or lung); the other 2 were peritoneal deposits biopsied during the reversal of a temporary ostomy.

Patients with RD detected outside scheduled FU did not miss more FU consultations or examinations than those with RD detected during scheduled FU visits $(p=0.16)$.

Two patients in whom RD was detected outside of scheduled FU were treated with curative intent (22\%) for a solitary liver and lung metastasis, respectively. Palliative care was started in the other 7 patients $(78 \%)$.

\section{Survival}

During the FU period, 22 patients died: 17 patients after being diagnosed with $\mathrm{RD}$ and 5 patients without $\mathrm{RD}$ due to other causes. Recurrence of rectal carcinoma was the direct cause of death in 14 patients. The estimated 5 -year overall survival rate was $85 \%$. The estimated recurrence free 5 -year survival was $77 \%$. Patients with RD detected during scheduled FU consultations had a significantly ( $p=0.001)$ higher survival rate than if $\mathrm{RD}$ was diagnosed during an interval consultation (Fig. 1). The median FU time after diagnosis with RD was 18 months (IQR 8-35).

\section{Discussion}

The purpose of this study was to identify when and how recurrences of rectal carcinoma were detected during the 5-year FU period after treatment with curative intent in order to assess whether future FU could possibly be transferred to primary care. Most of the 378 patients included in our study received regular FU consultations according to the national guideline. The 64 patients (17\%) who were diagnosed with $\mathrm{RD}$ in our study were mostly detected during scheduled FU and were asymptomatic. The median time until detection was 15 months, which is comparable to the 14 months reported for colon cancer recurrence by our study group [15]. Only 2 patients in this study were diagnosed with $\mathrm{RD}$ more than 3 years after surgery - just over $1 \%$ of patients with more than 3 years of FU. In this context, one could consider ending rectal cancer FU after 3 years, especially since the benefit of intensive FU has been questioned [5], and potential harmful side-effects of FU possibly been underestimated [16].

Previous studies reported locoregional recurrence rates of $2-15 \%$, and overall recurrence rates of $20-30 \%$

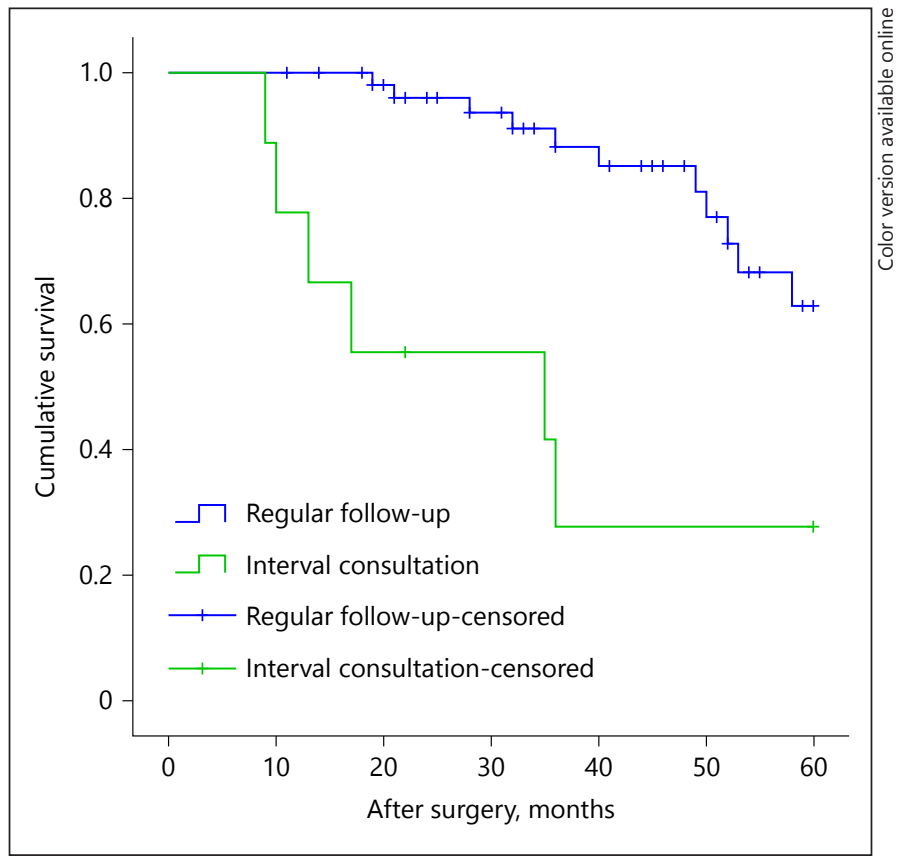

Fig. 1. Survival rate comparison regular FU versus interval consultation. Five-year overall survival rate of patients with recurrence of rectal carcinoma detected during regular follow-up versus detection at interval consultations, $p=0.001$.

[6-8, 17-22], which complies with the estimated 5-year recurrence-free survival rate of $77 \%$ found in our study. The variance in locoregional recurrence rates is often explained by the quality of surgery according to TME principles and the use of neoadjuvant RT $[19,20]$. In our study, $83 \%$ of patients received neoadjuvant RT; besides surgical quality, this could explain the relatively low percentage (3\%) of locoregional recurrences we found. Furthermore, we excluded all patients with stage IV disease, whereas most other studies included stage IV patients if they were treated with curative intent $[7,8,18,19,22]$. This may explain why the overall recurrence rate in this study is relatively low compared to previous studies.

While relatively few patients missed 2 or more blood tests or radiological examinations, nearly one in 3 patients did not have a surveillance colonoscopy. These findings differ from the results of the study conducted by Sisler et al. [23], who reported a concordance of colonoscopies with the Canadian national guideline of $80 \%$ within 3 years. Most colonoscopies in that study were performed by surgeons, while in the Netherlands a patient needs to be referred to a gastroenterologist for a colonoscopy, which might explain the difference in colonoscopy surveillance rates. The function of colonoscopy is mainly 
to detect metachronous tumours and inspect the anastomosis for local recurrence ( $5 \%$ of recurrences in this study was located at the anastomosis). In this way, it complements the other FU examinations and warrants continued recommendation. Recurrences detected during scheduled consultations were mostly found because of abnormal imaging, rising CEA blood levels or a combination of both. Accordingly, although there seems to be room for improvement in conducting a surveillance colonoscopy, the FU schedule seems adequate in detecting $\mathrm{RD}$ at an asymptomatic stage.

Half of patients with RD had a CEA level increase, which corresponds with Grossmann et al. [6], who found a rise in postoperative CEA levels in $41 \%$ of patients with $\mathrm{RD}$. Performing an accurate comparison of efficiency of radiological examination with previous reports is not feasible due to the heterogeneity of FU schedules $[4,5,7,22]$ or because treatment did not include neoadjuvant RT [24].

The few patients detected outside of scheduled FU consultations were more likely to present with symptoms, and less likely to receive treatment with curative intent. In our study, the low number of symptomatic patients $(n=$ 6) could be a consequence of the intensity of the FU schedule in the Netherlands. Previous studies reporting locoregional recurrence rates between 55 and $86 \%$ or a higher number of symptomatic patients were able to publish a more extensive list of symptoms associated with locoregional $\mathrm{RD}$, such as pain, anal discharge or blood loss, changed bowel habits or obstruction, urogenital problems, a palpable lump and weight-loss [22, 25-28].

\section{Strengths and Limitations}

This study systematically studied patients with stages I-III rectal carcinoma after curative treatment, and any subsequent $\mathrm{RD}$, thereby creating a clear overview of current rectal cancer FU. To our knowledge, this is the first study that evaluated the presentation of both locoregional and distant metastases in relation to scheduled FU.

Because the analysis was performed retrospectively, we were limited in our classification of symptoms by the documentation of the involved physician, possibly resulting in an underestimation of actual symptoms. The median FU time of 30 months is relatively short for a surveillance outcome study, although we feel it is unlikely that longer FU would have changed our results, especially since most recurrences occur within 3 years. The difference in detection of RD between scheduled and interval consultations is based on 9 patients and should therefore be interpreted with caution. Lastly, any possible current involvement of GPs in the detection of RD is unknown.

\section{Conclusions}

A majority of recurrences after curative treatment for rectal carcinoma were detected during scheduled FU consultations by means of standard examinations with nearly all recurrences being detected within 3 years after surgery. Despite the low number of symptomatic patients in our study, the difference in treatment options and survival between patients diagnosed with RD during or outside of a scheduled FU consultation is obvious. Our results encourage further exploration into a more prominent role of GPs in future rectal cancer aftercare; seeing as how most recurrences were detected with standard examinations during scheduled consultations. Can GPs execute the FU schedule in a similar way, while also providing comprehensive care (such as psychosocial support and attention to comorbid conditions)? The answer to this question needs to be the subject of future studies.

\section{Disclosure Statement}

The authors declare that they have no conflicting interests.

\section{Statement of Ethics}

The Research Ethics Committee of the AMC reviewed the protocol and assessed that the Medical Research Involving Human Subjects Act does not apply to this study. An official approval by the committee was therefore not required and written informed consent was not obtained.
References
1 The Dutch Cancer Registry: Comprehensive Cancer Centre the Netherlands (IKNL) (accessed February 24, 2016).

2 Oncoline: National Guideline Colorectalcarcinoma 2014 (accessed February 24, 2016).

3 Marijnen CAM, Vegchel TV: Nieuwe multidisciplinaire richtlijn voor de behandeling van het colorectaal carcinoom en colorectale levermetastasen. Nederlands Tijdschrift Voor Oncologie 2014;11.

4 Jeffery M, Hickey BE, Hider PN: Follow-up strategies for patients treated for non-metastatic colorectal cancer. Cochrane Database Syst Rev 2007;1:CD002200.

5 Primrose JN, Perera R, Gray A, Rose P, Fuller A, Corkhill A, et al: Effect of 3 to 5 years of scheduled CEA and CT follow-up to detect recurrence of colorectal cancer: the FACS randomized clinical trial. JAMA 2014;311: 263-270.
Wieldraaijer/Bruin/Duineveld/Tanis/ Smits/van Weert/Wind 
6 Grossmann I, de Bock GH, Meershoek-Klein Kranenbarg WM, van de Velde CJ, Wiggers $\mathrm{T}$ : Carcinoembryonic antigen (CEA) measurement during follow-up for rectal carcino$\mathrm{ma}$ is useful even if normal levels exist before surgery. A retrospective study of CEA values in the TME trial. Eur J Surg Oncol 2007;33: 183-187.

7 Rasanen M, Carpelan-Holmstrom M, Mustonen $\mathrm{H}$, Renkonen-Sinisalo L, Lepisto A: Pattern of rectal cancer recurrence after curative surgery. Int J Colorectal Dis 2015;30:775785.

8 Krivokapic Z, Barisic G, Markovic V, Popovic M, Antic S, Jovanovic D, et al: First thousand rectal cancer cases - local recurrence and survival. Acta Chir Iugosl 2004;51:133-137.

9 Tominaga T, Sakabe T, Koyama Y, Hamano K, Yasutomi M, Takahashi T, et al: Prognostic factors for patients with colon or rectal carcinoma treated with resection only. Fiveyear follow-up report. Cancer 1996;78:403408.

10 Health Council of the Netherlands: Followup in Oncology. Identify Objectives, Substantiate Actions. The Hague, Health Council of the Netherlands, 2007.

11 Dutch Cancer Society's Signalling Committee on Cancer (SCK of KWF Kankerbestrijding). Aftercare in Cancer, The Role of Primary Care, 2001

12 Wind J, Duineveld LA, van der Heijden RP, van Asselt KM, Bemelman WA, van Weert HC: Follow-up after colon cancer treatment in the Netherlands; a survey of patients, GPs, and colorectal surgeons. Eur J Surg Oncol 2013;39:837-843.
13 Augestad KM, Norum J, Dehof S, Aspevik R, Ringberg U, Nestvold T, et al: Cost-effectiveness and quality of life in surgeon versus general practitioner-organised colon cancer surveillance: a randomised controlled trial. BMJ Open 2013;3:pii:e002391.

14 Wattchow DA, Weller DP, Esterman A, Pilotto LS, McGorm K, Hammett Z, et al: General practice vs surgical-based follow-up for patients with colon cancer: randomised controlled trial. Br J Cancer 2006;94:1116-1121.

15 Duineveld LA, van Asselt KM, Bemelman WA, Smits AB, Tanis PJ, van Weert HC, et al: Symptomatic and asymptomatic colon cancer recurrence: a multicenter cohort study. Ann Fam Med 2016;14:215-220.

16 Augestad KM, Rose J, Crawshaw B, Cooper G, Delaney C: Do the benefits outweigh the side effects of colorectal cancer surveillance? A systematic review. World J Gastrointest Oncol 2014;6:104-111.

17 Yun HR, Lee LJ, Park JH, Cho YK, Cho YB, Lee WY, et al: Local recurrence after curative resection in patients with colon and rectal cancers. Int J Colorectal Dis 2008;23:10811087.

18 Kim NK, Kim YW, Min BS, Lee KY, Sohn SK, Cho $\mathrm{CH}$ : Factors associated with local recurrence after neoadjuvant chemoradiation with total mesorectal excision for rectal cancer. World J Surg 2009;33:1741-1749.

19 Jorgren F, Johansson R, Damber L, Lindmark G: Risk factors of rectal cancer local recurrence: population-based survey and validation of the Swedish rectal cancer registry. Colorectal Dis 2010;12:977-986.

20 Boras Z, Kondza G, Sisljagic V, Busic Z, Gmajnic R, Istvanic T: Prognostic factors of local recurrence and survival after curative rectal cancer surgery: a single institution experience. Coll Antropol 2012;36:1355-1361.
21 Ding P, Liska D, Tang P, Shia J, Saltz L, Goodman K, et al: Pulmonary recurrence predominates after combined modality therapy for rectal cancer: an original retrospective study. Ann Surg 2012;256:111-116.

22 Kodeda K, Derwinger K, Gustavsson B, Nordgren S: Local recurrence of rectal cancer: a population-based cohort study of diagnosis, treatment and outcome. Colorectal Dis 2012; 14:e230-e237.

23 Sisler JJ, Seo B, Katz A, Shu E, Chateau D, Czaykowski P, et al: Concordance with ASCO guidelines for surveillance after colorectal cancer treatment: a population-based analysis. J Oncol Pract 2012;8:e69-e79.

24 Secco GB, Fardelli R, Rovida S, Gianquinto D, Baldi E, Bonfante $\mathrm{P}$, et al: Is intensive followup really able to improve prognosis of patients with local recurrence after curative surgery for rectal cancer? Ann Surg Oncol 2000;7:32-37.

25 Salo JC, Paty PB, Guillem J, Minsky BD, Harrison LB, Cohen AM: Surgical salvage of recurrent rectal carcinoma after curative resection: a 10-year experience. Ann Surg Oncol 1999;6:171-177.

26 Bakx R, van Tinteren H, van Lanschot JJ, Zoetmulder FA: Surgical treatment of locally recurrent rectal cancer. Eur J Surg Oncol 2004;30:857-863.

27 Boyle KM, Sagar PM, Chalmers AG, SebagMontefiore D, Cairns A, Eardley I: Surgery for locally recurrent rectal cancer. Dis Colon Rectum 2005;48:929-937.

28 Heriot AG, Byrne CM, Lee P, Dobbs B, Tilney $\mathrm{H}$, Solomon MJ, et al: Extended radical resection: the choice for locally recurrent rectal cancer. Dis Colon Rectum 2008;51:284-291.
Clinical Pattern of RD during FU of Rectal Carcinoma 